\title{
Acute myocardial infarction with st-segment elevation: to aspirate or not?
}

\begin{abstract}
Acute myocardial infarction with ST-segment elevation (STEMI) is the most serious clinical manifestation of acute coronary syndrome (ACS). Golden therapeutic approach for patients with STEMI is primary percutaneous coronary intervention (PCI) and its aim is to restore normal coronary flow and provide myocardial tissue reperfusion as soon as possible leading to myocardial salvage. Thrombus aspiration is an adjunctive therapeutic modality to conventional PCI which can be especially powerful and helpful in some clinical subsets of STEMI with large thrombus burden. Here we discuss about advantages and disadvantages of the thrombus aspiration technique in regard to the most important clinical trials.
\end{abstract}

Keywords: STEMI, PCI, thrombus aspiration, reperfusion
Volume 8 Issue 3 - 2017

\author{
Marjan Boshev,' Savetka Paljoshkovska \\ Jordanova ${ }^{2}$ \\ 'Department for Invasive and Interventional Cardiology, \\ University Clinic of Cardiology, Macedonia \\ ${ }^{2}$ Department for Coronary Artery Disease, University Clinic of \\ Cardiology, Macedonia
}

\author{
Correspondence: Marjan Boshev, University Clinic of \\ Cardiology, Department for Invasive and Interventional \\ Cardiology, University Campus Mother Theresa, 1000 Skopje, \\ Republic of Macedonia, Tel 38977540808 , \\ Email marjanboshev@gmail.com
}

Received: February 26, 2017 | Published: March 17, 2017
Abbreviations: STEMI, ST-segment elevation myocardial infarction; ACS, acute coronary syndrome; PCI, percutaneous coronary interevention; TIMI, thrombolysis in myocardial infarction; MVO, microvascular obstruction; MBG, myocardial blush grade; STr, ST-segment resolution; CE-MRI, contrast-enhanced magnetic resonance imaging

\section{Introduction}

Acute myocardial infarction with ST-segment elevation (STEMI) is the most serious and life-threatening clinical manifestation of acute coronary syndrome (ACS) which can be treated pharmacologically (with fibrinolytic therapy) or interventionally (with percutaneous coronary intervention - PCI). Today primary PCI is a golden therapeutic approach for patients with STEMI $^{1}$ and it provides Thrombolysis In Myocardial Infarction (TIMI) flow grade 3 in the vast majority of patients. ${ }^{2}$

\section{Discussion}

Atherosclerotic plaque disruption with subsequent platelet activation as a result of direct exposure of the subintimal tissue to the bloodstream is the proposed pathophysiologic mechanism of intracoronary thrombus formation causing coronary flow obstruction. ${ }^{3}$ Thrombus consists of platelet aggregates, fibrin deposits, red blood cells as well as inflammatory substances (cytokines) and its composition may vary. Large thrombus burden very often may be a significant issue for conventional PCI. One of the most important parameters for interventional cardiologists in clinical setting of STEMI is recanalization (reperfusion) of the occluded coronary artery and restoration of the normal coronary flow as early as possible after chest pain onset. Thrombus aspiration is an established treatment modality in patients with STEMI presenting with large thrombus burden on coronary angiography (Figure 1). ${ }^{4}$ In most cases this technique can efficiently remove intracoronary thrombus and easily restore normal coronary flow (achieving TIMI flow grade 3 ).

Nevertheless, restoration of the normal coronary flow in the infarct-related epicardial coronary artery does not necessarily mean successful myocardial salvage. Namely, sometimes due to intracoronary manipulation with the thrombus aspiration catheter itself or during balloon inflation or stent deployment in conventional PCI procedures, there is an increased risk for distal embolization of thrombotic material leading to slow flow or no reflow phenomenon in distal artery branches (microvascular obstruction - MVO) and subsequent infarct extension. ${ }^{5,7,9}$ Thus, besides achieving intracoronary TIMI flow 3, very important issue in management of STEMI is providing optimal myocardial perfusion as well which can be assessed by so-called myocardial blush grade (MBG). In addition, there are some data that suggest that patients with TIMI flow grade 3 but with suboptimal MBG have worse clinical outcome, increased infarct size, higher incidence of heart failure and increased total mortality. ${ }^{6,8}$

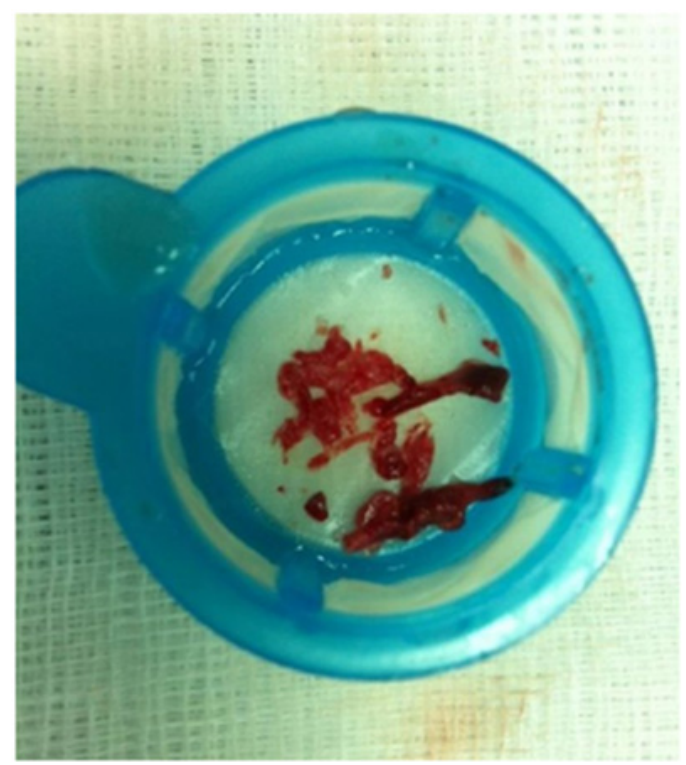

Figure I Thrombotic material evacuated from the coronary artery in patient with STEMI by using manual aspiration thrombectomy.

Another principal strategy to reduce thrombus burden is intracoronary/intravenous administration of glycoprotein IIb/IIIa inhibitors (GPIIb/IIIa inhibitors), especially as an adjunctive therapy to thrombus aspiration technique. Although effective, their routine use in STEMI patients is not recommended. ${ }^{3}$ Thrombus aspiration 
technique is relatively cheap and easy technique and generally can be performed by mechanical or manual thrombectomy devices. There are several clinical trials which deal with aspects of thrombus aspiration technique.

AiMI Trial (AngioJet in Acute Myocardial Infarction) was a multicenter, prospective, randomized trial comparing AngioJet thrombectomy followed by definitive treatment (PCI) versus immediate definitive treatment (PCI) only in patients with AMI undergoing PCI. Results from this trial showed that patients treated with rheolytic thrombectomy had increased infarct size and mortality rates in comparison with primary PCI control arm, which lead to the conclusion that routine use of rheolytic thrombectomy in STEMI patients is not recommended. ${ }^{10}$

X AMINE ST Trial (X-Sizer for Thrombectomy in Acute Myocardial Infarction Improves ST-Segmtn Resolution) was another prospective randomized multicenter study which compared the effect of adjunctive thrombectomy using X-Sizer befor PCI versus conventional PCI in patients with AMI in terms of ST-segment resolution after PCI. Summary results showed that use of X-Sizer catheter has improved myocardial reperfusion in patients with TIMI flow grade 0 to $1.3,9$

There are also clinical trials which are analyzing the impact of manual aspiration thrombectomy in STEMI patients. The REMEDIA trial for example, a prospective randomized trial, suggested that manual thrombus aspiration (with Diver CE device) in unselected patients with STEMI undergoing primary or rescue PCI was feasible and resulted in improved myocardial reperfusion. ${ }^{3,11}$ The DEAR-MI trial (Dethrombosis to Enhance Acute Reperfusion in Myocardial Infarction) showed that manual thrombus aspiration (with Pronto aspiration catheter) before primary PCI improved myocardial reperfusion and reduced rates of distal embolization and no reflow compared with conventional primary PCI. ${ }^{3,5}$ Similarly, the EXPIRA trial, a prospective randomized trial was designed to compare primary PCI alone versus primary PCI with manual thrombectomy in STEMI patients in terms of MBG and ST-segment resolution (STr), MVO and infarct size. It showed a significant improvement in the rates of MBG and STr in primary PCI with manual thrombectomy group. What was more interesting in this study was that it evaluated MVO and infarct size by using contrast-enhanced magnetic resonance imaging (CEMRI), a very useful tool for clinical trials., ${ }^{2,3}$

In line with these "positive" studies was also the TAPAS trial, a single-center, prospective randomized trial including 1071 patients, randomized to either manual aspiration thrombectomy with $6 \mathrm{~F}$ Export catheter before conventional PCI or conventional PCI alone. Primary endpoint was MBG whereas secondary endpoints were $\mathrm{STr}$ and clinical outcomes at 30days and 1 year. Results confirmed improvement in myocardial perfusion (MBG) and ECG markers of successful reperfusion (STr) after adjunctive thrombectomy. In addition, cardiac and all-cause mortality at 1-year were significantly reduced in the thrombectomy group..$^{3,4,6,12}$ Unfortunately, these positive results for manual aspiration thrombectomy were soon compromised by the results from other trials like the INFUSE-AMI, the TASTE and the TOTAL trial. The INFUSE-AMI trial (Intracoronary Abciximab and Aspiration Thrombectomy in Patients with Large Anterior Myocardial Infarction), a multicenter, randomized, single-blinded trial included 452 patients with anterior STEMI, randomized to either aspiration thrombectomy with Export catheter or conventional PCI only, as well as to intralesion abciximab vs no abciximab. This study failed to demonstrate a benefit of the manual thrombus aspiration on infarct size assessed by cardiac MRI. ${ }^{3,4,6}$
The TASTE trial (Thrombus Aspiration in ST-Elevation Myocardial Infarction in Scandinavia) was a multicenter, prospective, randomized, controlled, open-label clinical trial which randomized 7244 patients with STEMI to manual thrombus aspiration followed by PCI versus conventional PCI alone. Investigators of this study found that there was no significant benefit of aspiration thrombectomy in terms of mortality and any other clinical outcome at 30days., ${ }^{43}$ The largest and most important trial up to date regarding manual thrombus aspiration is the TOTAL trial (Trial of Routine Aspiration Thrombectomy with PCI Versus PCI Alone). The TOTAL trial recruited 10732 patients with acute STEMI and they were randomly assigned to a routine upfront manual thrombectomy versus PCI alone. Results from this study were somewhat disappointing because routine manual thrombectomy in comparison to PCI alone did not reduce the risk of $\mathrm{CV}$ death, recurrent myocardial infarction, cardiogenic shock, or NYHA class IV heart failure within 180days. Furthermore, investigators observed increased rate of stroke within 30days in the group of patients treated with manual thrombectomy. ${ }^{4,12}$

According to Hoole and colleagues, it seems that manipulation within the culprit lesion, regardless of whether balloon predilation or thrombectomy device is used first, increases the risk for distal embolization and MVO. ${ }^{7}$ In other words, it appears that management of the culprit lesion in STEMI patients should be "as simple as possible" and "as short as possible" which might favour primary direct stenting in order to prevent distal embolization. Currently available ESC guideline for management of STEMI (2012) recommends that thrombus aspiration should be considered as a routine procedure in primary PCI (class IIa, level B).${ }^{14}$ ACCF/AHA in 2013 released a guideline for management of STEMI and its recommendation is that manual aspiration thrombectomy is reasonable for patients undergoing primary PCI (class IIa, level of evidence B). ${ }^{15}$ But recently ACC/AHA/SCAI published a 2015 Focus Update on Primary PCI for Patients with STEMI and this document clearly emphasizes that "the usefulness of selective and bailout aspiration thrombectomy in patients undergoing primary PCI is not well established" (class IIb only). Furthermore, it includes a new recommendation which says that "routine aspiration thrombectomy before primary PCI is not useful" (class III - no benefit). ${ }^{16} \mathrm{We}$ are expecting the ESC this year (2017) to release an update for the guideline on AMI-STEMI treatment.

\section{Conclusion}

At the end, taking into consideration the results from previously mentioned clinical trials as well as current recommendations of the ESC and ACC/AHA/SCAI, we can summarize that manual aspiration thrombectomy is a simple, cheap, fast and powerful tool in the hands of interventional cardiologists which is not recommended as a routine procedure prior to PCI in patients with STEMI, but rather as an treatment option in highly selected patients with STEMI (large thrombus burden). Since the TOTAL study has reported that there was an increased risk for stroke within the first 30days in patients treated with manual aspiration thrombectomy, we can strongly recommend deep guiding catheter intubation (canulation) into the coronary ostium during thrombus aspiration process to prevent embolization in the systemic and cerebral circulation.

\section{Acknowledgments}

None.

\section{Conflicts of interest}

Author declares there is no conflicts of interest. 


\section{Funding}

None.

\section{References}

1. Fröbert, Lagerqvist B, Gudnason T, et al. Thrombus Aspiration in ST-Elevation myocardial infarction in Scandinavia (TASTE trial) A multicenter, prospective, randomized, controlled clinical registry trial based on the Swedish angiography and angioplasty registry (SCAAR) platform. Study design and rationale. Am Heart $J$. 2010;160(6):1042-1048.

2. Sardella, Mancone M, Bucciarelli-Ducci C, Agati L, et al. Thrombus Aspiration During Primary Percutaneous Coronary Intervention Improves Myocardial Reperfusion and Reduces Infarct Size. The EXPIRA(Thrombectomy With Export Catheter in Infarct-Related Artery During Primary Percutaneous Coronary Intervention) Prospective, Randomized Trial. J Am Coll Cardiol. 2009;53(4):309-315.

3. Nahlawi G, Brener J S. Thrombus aspiration in acute myocardial infarction. Interv Cardiol. 2013;5(6):673-681.

4. Desch S, Stiermaier T, De Waha S, et al. Thrombus Aspiration in Patients With ST-Segment Elevation Myocardial Infarction Presenting Late After Symptom Onset. JACC Cardiovasc Interv. 2016;9(2):113-122.

5. Silva Orrego, Colombo P, Gregorgi, et al. Thrombus Aspiration Before Primary Angioplasty Improves Myocardial Reperfusion in Acute Myocardial Infarction The DEAR-MI (Dethrombosis to Enhance Acute Reperfusion in Myocardial Infarction) Study. J Am Coll Cardiol. 2006;48(8):1552-1559.

6. Hacioglu Y, Ahmed Z, Hakeem A, et al. Manual Thrombus Aspiration in STEMI Treatment. Cardiac Interventions. 2013;501:257-5795.

7. Fröhlich GM, Landmesser U. Thrombus aspiration in STEMI revisited: impact on coronary microcirculation?. Open Heart. 2015;2(1):e000274.
8. Ikari, Sakurada, Kozuma, et al. Upfront Thrombus Aspiration in Primary Coronary Intervention for Patients with ST-Segment Elevation Acute Myocardial Infarction. Report of the VAMPIRE (VAcuuM asPIration thrombus REmoval) Trial. JACC Cardiovasc Interv. 2008;1(4):424-431.

9. Lefèvre, Garcia, Reimers, et al. X-Sizer for Thrombectomy in Acute Myocardial Infarction Improves ST-Segment Resolution Results of the X-Sizer in AMI for Negligible Embolization and Optimal ST Resolution (X AMINE ST) Trial. J Am Coll Cardiol . 2005;46(2):246-252.

10. Ali, Cox D, Dib N, et al. Rheolytic Thrombectomy With Percutaneous Coronary Intervention for Infarct Size Reduction in Acute Myocardial Infarction (AiMI Trial). J Am Coll Cardiol . 2006;48(2):244-252.

11. Burzotta, Carlo Trani, Enrico, , et al. Manual Thrombus-Aspiration Improves Myocardial Reperfusion (REMEDIA Trial). JACC. 2005;46(2):371-376.

12. Jolly, John, Salim, et al. Randomized Trial of Primary PCI with or without Routine Manual Thrombectomy. N Engl J Med. 2015;372:1389-1398.

13. Fröbert, Bo Lagerqvist, Goran, et al. Thrombus Aspiration during ST-Segment Elevation Myocardial Infarction. $N$ Engl $J$ Med. 2013;369:1587-1597.

14. Stefan, Dan Atar, Luigi, et al. ESC Guidelines for the management of acute myocardial infarction in patients presenting with ST-segment elevation: The Task Force on the management of ST-segment elevation acute myocardial infarction of the European Society of Cardiology (ESC). European Heart Journal. 2012;33(20):2569-2619.

15. O'Gara, Frederick GK, Deborah DA, et al. 2013 ACCF/AHA Guideline for the Management of ST-Elevation Myocardial Infarction: Executive Summary JACC. 2013;61(4):1-508.

16. Levine, Eric RB, James CB, et al. 2015 ACC/AHA/SCAI Focused Update on Primary Percutaneous Coronary Intervention for patients With ST-Elevation Myocardial Infarction. JACC . 2016;67(10):1236-1247. 